\title{
Hacia una teoría sociocrítica del texto*
}

Fecha de recepción: 2 de Julio de 2017

Fecha de aprobación: 9 de septiembre de 2017

\section{Resumen}

El análisis de la microsemiótica intratextual permite observar las contradicciones resultantes de las brechas históricas proyectadas en los textos literarios, los cuales reproducen, a su vez, las contradicciones de las estructuras sociales e ideológicas. En su modelo, Cros privilegia un espacio intratextual abstracto que denomina genotexto, a través del cual se incorporan la historia y sus contradicciones. El material histórico invertido en el genotexto corresponde a los principales intereses de una sociedad en un momento dado de su historia, cuestión que implica que el escritor da la palabra en su obra, aunque no sea de un modo consciente, a una serie de sujetos transindividuales que lo atraviesan. Esto se ilustra a través del análisis de un apartado del segundo volumen de Guzmán de Alfarache, de Mateo Alemán (Sevilla, 1547 - México, 1614), editado en 1604.

Palabras clave: sociocrítica, genotexto, fenotexto, literatura-historia, sujeto transindividual, Guzmán de Alfarache.

Citar: Escobar, H., Juliana, B. (julio-diciembre de 2017). Hacia una teoría sociocrítica del texto. Edmond Cros (traducción de Hernando Escobar y Juliana Borrero). La Palabra, (31), 29-38. doi: https://doi.org/10.19053/01218530.n31.2017.7272.

\section{Edmond Cros}

Catedrático emérito de la Universidad "Paul Valéry"- Montepellier III (Francia)

Traducido por Hernando Escobar-Vera y Juliana Borrero Echeverry

Docentes de la Maestría en Literatura. Investigadores del grupo Senderos del Lenguaje, Universidad Pedagógica y Tecnológica de Colombia

*Artículo publicado originalmente en inglés en Hansen, Hans Lauge (ed.) (2006). Disciplines and Interdisciplinarity in Foreign Language Studies. Copenague: Museum Tusculanum Press - Universidad de Copenague, pp. 121-130. También, en 2011, en la revista Sociocriticism (Universidad de Granada), 26 (1 y 2), pp. 31-47, disponible en: http://revistaseug.ugr.es/index.php/sociocriticism/article/ view/2452/2567. La presente traducción se basa en la edición de Sociocriticism; fue revisada por el autor e incorpora sus precisiones.

Esta traducción es producto de investigación del grupo Senderos del Lenguaje, de la Universidad Pedagógica y Tecnológica de Colombia. 


\section{la palabra \\ Towards a Sociocritical Theory of the Text}

\section{Abstract}

The analysis of intratextual microsemiotics allows for the observation of contradictions resulting from the historical gaps projected in literary texts, which in turn reproduce the contradictions present in social and ideological structures. In his model, Cros privileges an abstract intratextual space named genotext, through which history and its contradictions are incorporated. The historical material invested in the genotext corresponds to the principal interests of a society in a given moment of history. This implies that in the text, the writer gives word, even if non-consciously, to a series of transindividual subjects that run through it. This is illustrated through the analysis of a fragment of the second volume of Guzmán de Alfarache, by Mateo Alemán (Sevilla, 1547 - México, 1614), edited in 1604.

Keywords: sociocriticism, genotext, phenotext, literature-history relation, transindividual subject, Guzmán de Alfarache.

\section{Vers une théorie sociocritique du texte}

\section{Résumé}

L'analyse de la microsémiotique intratextuelle nous permet de mettre en évidence les contradictions produites par les fissures historiques projetées sur les textes littéraires, qui reproduisent, à leur tour, les contradictions des structures sociales et idélogiques. Dans son modele, Cros privilégie un espace intratextuel abstrait qu'il appelle génotexte oú l'histoire et ses contradictions sont intégrées. Le matériel historique investit dans le génotexte correspond aux principaux intérêts d'une société à un moment donné de son histoire. Ainsi, l'ecrivain donne la parole, bien que de manière inconsciente, à plusieurs sujets transindividuels qui traversent le texte. Nous illustrerons ceci à partir de l'analyse d'une partie du deuxième volume de Guzmán de Alfarache, de Mateo Alemán (Seville, 1547 - Méxique, 1614), edité en 1604 .

Mots-clés: sociocritique, génotexte, phénotexte, littérature-histoire, sujet transindividuel, Guzmán de Alfarache. 


\section{Introducción}

En los años sesenta, observamos una reconfiguración radical del concepto de texto, resultante de la rápida expansión de las semiologías generales lingüísticas y literarias. Este concepto, independiente de la filosofía de la verdad, definía un nuevo objeto, que fue descrito como un dispositivo translingüístico $\mathrm{y}$ fue considerado como una práctica significante que nunca deja de operar y que es irreductible a alguna significación objetiva. En tanto retiene los conceptos teóricos implícitos en este concepto, la sociocrítica se ocupa esencialmente de aquello que el texto transcribe, es decir, de las modalidades de incorporación de la historia, no en el nivel del contenido, sino en el de la forma. Para la sociocrítica, la pluralidad es el resultado de los procesos dinámicos y dialécticos de la historia. De hecho, es debido a que incorpora la historia de una manera que le es propia, que el texto se presenta como un dispositivo translingüístico. Son estos caminos de sentido complejo, heterogéneo y contradictorio los que intento señalar e identificar tanto en su naturaleza, como en sus efectos.

La sociocrítica procura poner de manifiesto las relaciones existentes entre las estructuras de la obra literaria (o cultural) y las de la sociedad en la que está profundamente arraigada. Afirma que dar con las huellas ideológicas y las tensiones antagónicas entre las clases sociales es fundamental para cualquier lectura de textos.

A diferencia de la mayoría de enfoques sociológicos de la literatura que dejan intactas las estructuras del texto, la sociocrítica sienta que la naturaleza social de la obra literaria debe ser localizada e investigada dentro del texto y no fuera de él. Es necesario reconstruir de manera paciente y exacta los elementos semiótico-ideológicos, para mostrar cómo el proceso histórico está profundamente involucrado en el proceso de escritura. De hecho, es necesario examinar las diferentes formas en que la historia es incorporada en el texto. Sobre este punto, es preciso enfatizar en una serie de preguntas:

- ¿Qué tipo de material histórico nos interesa?

- ¿Cómo se supone que el texto incorpora este material histórico?

- ¿Qué enfoque teórico y metodológico permite en el ejercicio crítico sacar a la luz el proceso de la incorporación de la historia?

Antes de responder estas preguntas, debo destacar que toda teoría se fundamenta en dos puntos:
1. una concepción filosófica que implica un punto de vista respecto a la historia, con lo cual cuestiona cuál es su proceso,

2. una concepción poética referente al funcionamiento del texto.

Entonces, ¿cuál es el proceso de la historia?, ¿cómo definir lo que es el texto?, ¿cómo funciona el texto? Respecto al primer asunto, me refiero al marxismo, que vincula la formación discursiva con las formaciones ideológica y social. De hecho, existe una relación entre la infraestructura y la superestructura, pero esta relación no es automática ni directa. Entre los dos niveles (y dentro de ellos) es necesario distinguir una serie de instancias, pertenecientes cada una a tiempos históricos diferentes. En un momento dado de la historia, un sujeto determinado parece situado en una posición adelantada respecto a su tiempo, y otros parecen rezagados. En la medida en que el rezagado es siempre atraído por el adelantado, la brecha entre las dos posiciones y la serie de brechas existentes en la totalidad del sistema producen el dinamismo del proceso. Estas brechas históricas generan huellas semiótico-ideológicas y diversos tipos de efectos en la obra literaria, observables especialmente en los espacios textuales de las contradicciones. Por eso, en mi lectura crítica 
parto de las microsemióticas intratextuales que se organizan a partir de estas contradicciones, las cuales nos permiten reconstruir las formaciones sociales e ideológicas.

Ahora, ¿cómo funciona el texto? Cuando empieza a construirse, el texto empieza a establecer sus reglas de repetición: repite una corta serie de mensajes, pero no de manera monótona (o de manera exactamente igual); lo hace a través de los diferentes niveles o categorías de los textos (es decir: tiempo, espacio, material discursivo, mito, temas, etc., y cada texto puede presentar categorías específicas). Estos mensajes nacen de un espacio intratextual abstracto que denomino genotexto.

\section{Genotexto y fenotexto}

El genotexto es un campo semiótico que parece totalmente neutro , pero, al mismo tiempo, está cruzado por contradicciones ideológicas. Está constituido por un sistema combinatorio de elementos genéticos, responsables de la producción global de sentido y portadores del conflicto. Estos elementos funcionan de un modo pluri-acentuado, y afirmo que estas contradicciones reproducen las contradicciones de las formaciones sociales e ideológicas.

Pero el genotexto no existe en el texto: en el texto sólo tratamos con los fenotextos. Ya for- mulado el genotexto, nos consta que los fenotextos aparecen en todas las categorías del texto y cada categoría deconstruye el genotexto de acuerdo con las reglas específicas de su propio funcionamiento. La expresión del tiempo, por ejemplo, da un resultado, una actualización, muy diferente de la operada por la expresión del espacio.

Estos términos no se refieren a las nociones de Julia Kristeva (1969, pp. 280-283), sino que los tomo de la geografía humana. Para entender lo que quiero decir, es preciso recordar las nociones de fenotipo y genotipo. La mujer mediterránea es un genotipo, pero no existe; lo que sí existe son varias mujeres que viven en las diferentes orillas del Mar Mediterráneo, con características similares. A partir de (y por medio de) estas características hemos elaborado una figura abstracta.

El genotexto no es exactamente una estructura, sino que se convierte en una, estructurándose a través de las diferentes concreciones fenotextuales del mismo texto. En el fenotexto, la enunciación no gramaticalizada del genotexto y las características apropiadas en un nivel determinado funcionan en el marco de un proceso de significación que actualiza, de manera aparentemente incoherente y fragmentada, las latencias semánticas de la misma enunciación: el genotexto. Este existe solamente a través de estas elaboraciones múltiples y concretas -fenotextuales - y corresponde a una abstracción reconstruida por el analista.

\section{Genotexto e historia}

En la medida en que el genotexto es el medio a través del cual el texto incorpora la historia, podemos entender que los elementos incorporados, en forma de fuertes contradicciones, son los fundamentales, los que portan el porvenir de una sociedad dada y constituyen sus más importantes apuestas.

¿Cómo funciona el genotexto? ¿De dónde viene? Usando una metáfora espacial, podemos imaginar el punto de intersección de dos ejes, uno vertical y otro horizontal. En el primer eje está el interdiscurso, que materializa tanto las estructuras mentales como las formaciones ideológicas producidas por una formación social. El discurso del tiempo acerca de sí mismo se lee en este eje, es decir, el interdiscurso traduce a operaciones semióticas las condiciones socio-históricas en las que un hablante está inmerso. En el eje horizontal encontramos el intertexto (pre-afirmado, pre-construido, pre-condicionado), es decir, todo el material lingüístico destinado a dar forma al significado. 


\section{Cuadro 1.}

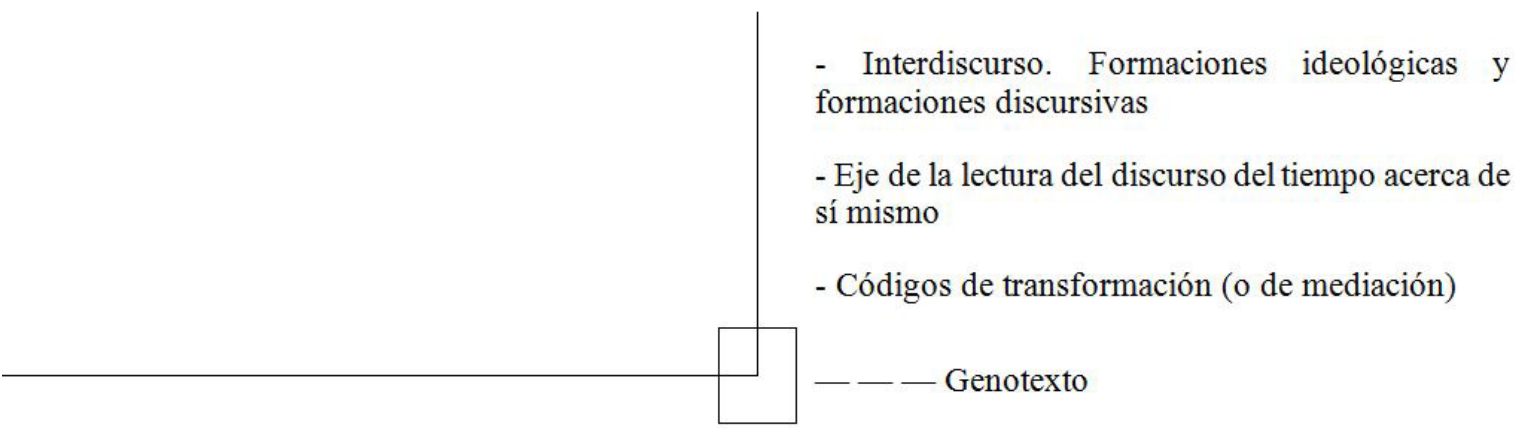

- Intertextualidad - constructos previos - precondicionamientos

- Eje de los sistemas modeladores

- Códigos simbólicos

\section{¿Qué es el interdiscurso?}

Para explicar lo que el término genotexto significa para mí, quisiera recordar dos nociones de Lucien Goldmann (1966): el sujeto transindividual (colectivo) y el nivel de lo no-consciente. Cada uno de nosotros pertenece, en algún momento de la vida, a una serie de sujetos colectivos (generación, familia, origen geográfico, profesión, etc.). Pasamos por muchos de estos en el curso de nuestra existencia. Estos diversos sujetos colectivos, al pasar por ellos, nos ofrecen sus valores sociales y visiones de mundo a través de sus discursos específicos. Cada sujeto transindividual inscribe en su discurso los signos de su inserción espacial, social e histórica y, en consecuen- cia, genera una microsemiótica específica.

La totalidad del material discursivo que usamos a lo largo de la vida se compone de este mosaico de discursos. Por eso, el texto no selecciona sus signos solamente dentro del lenguaje, sino entre la totalidad de las expresiones semióticas adquiridas / propuestas por los sujetos colectivos. Este sujeto transindividual se invierte ${ }^{1}$ en la conciencia de cada individuo que lo conforma, por medio de microsemióticas específicas. Estas microsemióticas transcriben en signos la totalidad de las aspiraciones, las frustraciones y los problemas vitales del grupo. Proporcionan una especie de decodificación de los modos en que cada grupo está inmerso.
Al reconstruir el nivel microsemiótico del texto, nos permitimos reconstruir la formación social en la que está inmerso el escritor.

La noción de Goldmann de sujeto transindividual requiere mayor precisión en la medida en que parece operar para él solo al nivel de los valores implícitos de una obra literaria. Es por eso que intenté describir sus efectos de una manera más precisa. Traté de describir los niveles en los que se pueden encontrar estos índices. Me parece que los rastros más obvios se encuentran en los ejes paradigmáticos, en las frases hechas y en las lexias. La forma en que se lexicalizan transcribe los sistemas de valores sociales. Los cambios que los modifican

Nota del traductor: la palabra usada por el autor en la versión inglesa del artículo es "invest". Él eligió "invertir" para la versión en español. 
transcriben los modos de vida y de inserción socioeconómica, así como la evolución de las estructuras mentales de los entornos que las producen.

Ahora, ¿cómo funciona u opera el discurso del sujeto transindividual? Goldmann distingue entre tres niveles diferentes de conciencia: a los primeros dos (inconsciente y conciencia despierta), él agrega lo no-consciente. Lo no-consciente es una creación del sujeto colectivo. Es diferente del inconsciente freudiano, por el hecho de que no está reprimido y, por tanto, no necesita superar ninguna resistencia para volverse consciente, pero solo puede salir a la luz a través del análisis científico. De hecho, al reproducir las prácticas sociales y discursivas de los sujetos colectivos, estamos diciendo mucho más de lo que sabemos o deseamos; generalmente estamos reproduciendo los valores sociales de diferentes sujetos colectivos. Ese es el espacio y el nivel del proceso genético que interesa a la sociocrítica.

Desde este punto de vista, podemos abordar mejor la siguiente pregunta básica: si bien la visibilidad social y personal del escritor es limitada, suponemos que la visibilidad de la obra literaria es a menudo mucho más amplia, ¿cómo explica el crítico esta diferencia? Esta diferencia es, para mí, el resultado del funcionamiento de lo no-cons- ciente. De hecho, más allá del campo de la visibilidad social propiamente dicha, se extiende otro campo interiorizado, aunque no conscientemente, responsable de las microsemióticas intratextuales. Estas microsemióticas reproducen los valores sociales de los diferentes sujetos colectivos a los que pertenece el escritor. Las relaciones con el mundo no son ni percibidas ni perceptibles en el nivel de la experiencia inmediata. Los diferentes discursos y los diferentes comportamientos del sujeto siempre tienen más significado del que el sujeto podría saber o desear. Este excedente de significado está almacenado en la microsemiótica intratextual, compuesta por el material semiótico no-consciente del sujeto colectivo implicado en el proceso de escritura. Al hacer que el sistema semiótico funcione por escrito, quien escribe siempre dice más de lo que entiende y más de lo que aparentemente afirma.

\section{A manera de ejemplo: una mi- rada a Guzmán de Alfarache}

Con el fin de hacer más clara esta presentación, sugiero una mirada rápida a un texto del Siglo de Oro español: la novela picaresca de Mateo Alemán Guzmán de Alfarache, editada entre 1599 y 1604.

Conforme a lo cual, siempre se tuvo por dificultoso hallarse un fiel amigo $y$ verdadero. Son contados, por escrito están y lo más en fábulas, los que se dice haberlo sido. Uno solo hallé de nuestra misma naturaleza, el mejor, el más liberal, verdadero $\mathrm{y}$ cierto de todos, que nunca falta y permanece, siempre sin cansarse de darnos; y es la tierra.

Esta nos da las piedras de precio, el oro, la plata y más metales, de que tanta necesidad y sed tenemos. Produce la yerba, con que no sólo se sustentan los ganados y animales de que nos valemos para cosas de nuestro servicio; mas juntamente aquellas medicinales, que nos conservan la salud y aligeran la enfermedad, preservándonos della. Cría nuestros frutos, dándonos telas con que cubrirnos y adornarnos. Rompe sus venas, brotando de sus pechos dulcísimas y misteriosas aguas que bebemos, arroyos y ríos que fertilizan los campos y facilitan los comercios, comunicándose por ellos las partes más extrañas y remotas. Todo nos lo consiente y sufre, bueno y mal tratamiento. A todo calla; es como la oveja, que nunca le oirán otra cosa que bien: si la llevan a comer, si a beber, si la encierran, si lo quitan 
el hijo, la lana y la vida, siempre a todo dice bien.

Y todo el bien que tenemos en la tierra, la tierra lo da. Úlimamente, ya después de fallecidos y hediondos, cuando no hay mujer, padre, hijo, pariente ni amigo que quiera sufrirnos y todos nos despiden, huyendo de nosotros, entonces nos ampara, recogiéndonos dentro de su propio vientre, donde nos guarda en fiel depósito, para volvernos a dar en vida nueva y eterna. Y la mayor excelencia, la más digna de Gloria y alabanza, es que, haciendo por nosotros tanto, tan a la continua, siendo tan generosa y franca, que ni cesa ni se cansa, nunca repite lo que da ni lo zahiere dando con ello en los ojos, como lo hacen los hombres. (Alemán, P.II, L.II, cap.1, edición de F. Rico, La novela picaresca, Barcelona, 1967).

Antes que nada, evoquemos brevemente la formación social en el Siglo de Oro español. Si tratamos de dar un panorama de los diversos intereses sociales, debemos señalar la próspera posición del comercio y de la asociación de grandes ganaderos reagrupados en La Mesta, a expensas de los fabricantes de telas y de los productores agrícolas. El gobierno de Carlos V fomenta la exportación de la mejor lana al norte de Europa e importa ropa que España luego exporta a las Indias. De esta manera, por ejemplo, el ganado tiene el privilegio de pasar a través de los campos cultivados del país, destruyéndolos. El texto de Alemán está escrito en el momento en que la avalancha de plata proveniente de América alcanzó su punto máximo, lo cual generó una fuerte polémica que opuso dos sistemas de pensamiento que han coexistido y luchado entre sí, respecto al papel del oro y los metales preciosos en la prosperidad de un Estado: ¿es el oro el "único signo de prosperidad individual o de la grandeza de un estado? $\mathrm{O}$, por el contrario, ¿es el comienzo de la disolución de la verdadera riqueza que consiste únicamente en la producción de bienes necesarios para la vida?" (Vilar, 1974, p.192). Desde este punto de vista, podemos comprender mejor la nueva contradicción entre la producción de bienes agrícolas e industriales y la acumulación de dinero (mediante el comercio o la importación de metales preciosos) como la mejor manera de crear prosperidad económica. De hecho, los intereses de los ganaderos están relacionados con el comercio y con las actividades de los banqueros.

Se supone que el texto examinado elogia al fiel y verdadero amigo que te da todo lo que posee sin pedir nada a cambio. Desarrolla un lugar común, un topos, el elogio de la fecundidad de la Tierra, el mito de la Edad de Oro, la vida del hombre primitivo en un mundo natural en el que la Naturaleza daba su riqueza de forma espontánea (Lucrecio, De natura rerum). El hombre solo tiene que extender las manos y recoger los frutos. No necesita trabajar. Este tema está asociado con la condena de la aventura, por tierra o por mar, con fines comerciales y de propiedad individual. A partir de esto se desvanecen cuestiones como el esfuerzo, el trabajo y la riqueza privada. Más tarde, con las Geórgicas de Virgilio, aparece otra formulación del mito que desarrolla la idea de que la Tierra es fecunda si está bien cultivada. Esta nueva formulación se vincula con la noción de progreso en la agricultura, gracias a la intervención bondadosa de los dioses. Recordemos que las Geórgicas fueron escritas como una petición del Mecenas, que apoyaba así el plan de Octavio para restaurar en el pueblo romano las antiguas virtudes de las razas, especialmente el gusto por la agricultura. Este tema crea en el seno de la primera formulación del mito un espacio de conflicto, en la medida en que traduce las mismas nociones (felicidad y virtud) a discursos figurativos contradictorios (esfuerzo contra holgazanería / propiedad privada contra colectivismo). Del De natura rerum a las Geórgicas, el lugar común del elogio a la Tierra cambia, pues, de un 
discurso ateísta a uno ético-religioso al servicio de un proyecto político.

El texto de Alemán opera en los intersticios de este lugar común. La miel y los frutos silvestres de las descripciones latinas han quedado fuera; solo queda la forma mucho más general de "fruto". Se agregan cuatro productos: metales, hierba, ropa, agua. De la hierba a la tela y a las ovejas se construye un movimiento panegírico en el que se glorifica la crianza. Observemos lo que ocurre con el agua, tradicionalmente vinculada a la vida ("sin agua ningún hombre, ningún otro animal puede sostener la vida"). Aquí, por el contrario, su principal mérito es permitir el comercio y la comunicación entre las personas más distantes del mundo. Esta perspectiva de las aventuras en el extranjero, que hace hincapié en la importancia del comercio internacional y la cría de animales, revela el punto de vista a partir del cual se reconfigura el topos. La interdicción del comercio, observable en todos los textos latinos, es transgredida y ocupa todo el espacio textual. El lugar común es completamente invertido.

Es por eso que la concisión de "dándonos telas" es notable: borra todo el proceso de trans- formación material. De hecho, ni el campo agrícola ni el industrial son presentados en el texto. Esta ausencia, esta brecha, reproduce obviamente las brechas en la formación social existentes entre distintos tiempos históricos.

Investiguemos ahora la escritura misma. Observo, en una primera lectura, algunos fenómenos de difracciones semánticas y semióticas o reconfiguraciones de lugares comunes:

1. El primero se refiere a "piedras de precio" (y no piedras preciosas como en la expresión común). Por encima de la formulación original (piedras preciosas) se ha superpuesto el concepto de intercambio monetario de precio, a expensas del sentido metafórico de los objetos que serían estimables en relación con otros criterios, por ejemplo, emocionales o estéticos.

2. Otra deconstrucción similar aparece en "fiel amigo y verdadero". La formulación usual es: "buen y verdadero amigo". El término español utilizado en este texto es muy interesante, en la medida en que el término fiel se refiere, por ejemplo, al sirviente que no roba a su amo y es, también, el nombre de las personas que constatan oficialmente los pesos y los precios de los productos en los mercados.

3. "Son contados". En el eje paradigmático, los adjetivos más ampliamente utilizados son raros, pocos, escasos. En este eje, el texto selecciona, de hecho, un término obviamente connotado de manera similar a los otros ejemplos que estamos mencionando.

4. Pero la deconstrucción más sorprendente se ofrece con la expresión: "donde nos guarda en fiel depósito". El traductor al inglés [Mabbe] entiende muy bien la oración, la desarrolla y la explica haciendo referencia a que la expresión española pertenece al vocabulario del mundo bancario ${ }^{2}$.

5. Podríamos agregar una serie de expresiones establecidas, pertenecientes al vocabulario de la ley comercial (en cursivas en el texto citado) como: "conforme a lo cual" y "por escrito están". ${ }^{3}$

El material semiótico del discurso se ve así como una representación del mundo de las transacciones consideradas junto con sus actividades, valores,

\footnotetext{
2 Nota del traductor: esta expresión es traducida al inglés por Mabbe (1623) como: "where we are in a safe bank deposit" [donde estamos en un depósito bancario seguro].

3 Nota del traductor: se ha omitido un numeral, en el que Cros compara el texto original, en español, y su traducción al inglés, por parte de Mabbe (1623).
} 
reglas de comportamiento $\mathrm{y}$ organización jurídica. Siguiendo de esta manera los marcadores textuales de un discurso dominante, se revela el sistema ideológico responsable de la deconstrucción de los topos.

\section{Conclusiones}

El discurso invertido en el texto y que opera como productor de la transformación se pone claramente de manifiesto: es el discurso de un sujeto colectivo identificable: el comerciante y el capitalismo mercantil, lo cual implica un tiempo histórico determinado. Este discurso genera el nivel microsemiótico que hemos señalado y reconstruido a través de la reconfiguración de los lugares comunes analizados. En este caso, el discurso implica un valor fundamental, el intercambio; es decir, lo contrario del regalo. Mientras el escritor afirma que nos está presentando el modelo del amigo perfecto, que da todo lo que posee $\sin$ pedir nada, obviamente está re- velando una visión del mundo muy contradictoria. Es por eso que puedo definir el elemento principal del genotexto como una contradicción entre dar e intercambiar.

Este funcionamiento se pone de manifiesto, al menos en mi análisis, en tres niveles o tres fenotextos: el tema explícito (la total generosidad del amigo fiel versus la categoría del material verbal utilizado para describirlo), el mito y el material discursivo en sí mismo. Un análisis más detallado trae a la vista más categorías textuales que funcionan de la misma manera: la problemática religiosa, por ejemplo, en el cuestionamiento de las relaciones entre el mérito humano (un intercambio entre los actos humanos y la salvación) y la Santa Bondad de Dios que nos concede la salvación sin verificar si la merecemos.

Finalmente, debo mencionar el compromiso social de Mateo Alemán, quien apoya la refor- ma de la mendicidad en España y aboga por que debemos dar limosnas solo a los pobres que no pueden trabajar. Esta nueva concepción introduce la noción de mérito $\mathrm{y}$, consecuentemente, la de intercambio de una manera contradictoria, puesto que la concepción católica tradicional no permite ningún límite a la caridad. Si confiamos en una carta que escribió a un amigo, Alemán compuso su libro para apoyar esta reforma social proveniente de los países protestantes de Europa, una reforma que provocó fuertes polémicas en España.

De este modo, podemos entender mejor que el material histórico invertido en el genotexto corresponde a los principales intereses de una sociedad en un momento determinado de su historia, y observamos que este material histórico es el vector del proceso dinámico de la producción textual.

\section{Referencias}

Alemán, Mateo (1967 [1604]). "Segunda parte de la vida de Guzmán de Alfarache, atalaya de la vida humana”. En Francisco Rico, ed., La novela picaresca española, vol. 1. Barcelona: Planeta.

Cros, Edmond (1971). Mateo Alemán. Introducción a su vida y a su obra. Madrid: Anaya.

Cros, Edmond (1988). Theory and Practice of Sociocriticism. Minneapolis: University of Minnesota Press. 
Goldmann, Lucien (1966). Sciences humaines et philosophie. Paris: Gonthier.

Kristeva, Julia (1969). Semiotiké. Recherches pour une sémanalyse. Paris: Seuil.

Lucretius (1937). De natura rerum. London: Heineman Loeb Clasical Library / Cambridge: Harvard University Press (tercera edición).

Vilar, Pierre (1974). Or et monnaie dans l'Histoire 1450-1920. Paris: Flammarion.

Virgil (1947). Ecloges. Georgies. Aenid. Londres/Nueva York: Loeb Classical Library. 